\title{
INFLUÊNCIA DA SOBRECARGA NA QUALIDADE SUBJETIVA DO SONO DE IDOSOS CUIDADORES DE IDOSOS
}

Marcela Naiara Graciani Fumagale Macedo; Universidade Federal de São Carlos; marcelagracianivest@hotmail.com

Sonia Goncalves da Mota; Universidade Federal de São Carlos; soniagmota_47@hotmail.com

Daniele Cristina Barbosa; Universidade Federal de São Carlos; danibarbosa926@gmail.com Isabela Bonifacio de Almeida Ferreira; Universidade Federal de São Carlos; isabelabaferreira@gmail.com Élen dos Santos Alves; Universidade Federal de São Carlos; elendutra23@gmail.com Keika Inouye; Universidade Federal de São Carlos; keikain@ufscar.br Ariene Angelini dos Santos Orlandi; Universidade Federal de São Carlos; arieneangelini@yahoo.com.br

\section{RESUMO}

Introdução: Com o envelhecimento populacional, ser idoso e ter como responsabilidade cuidar de outro idoso é uma condição cada vez mais frequente. As atribuições do cuidado podem gerar sobrecarga e distúrbios do sono. Objetivo: Identificar associação entre sobrecarga e qualidade subjetiva do sono de idosos cuidadores de idosos. Métodos: A amostra foi composta por idosos cuidadores de idosos cadastrados em cinco Unidades de Saúde da Família do município de São Carlos (SP). Foram utilizados os seguintes instrumentos: Questionário de Caracterização, Componente 1 do Índice de Qualidade de Sono de Pittsburg e Escala de Sobrecarga de Zarit. Os cuidadores foram divididos em dois grupos: (a) Grupo com boa/muito boa qualidade do sono e (b) Grupo com qualidade do sono ruim/muito ruim. Foram realizadas análises estatísticas descritivas e comparativas (Teste de Mann-Whitney). Resultados: Dos 59 idosos cuidadores de idosos entrevistados, 56,9\% eram do sexo feminino ( $\mathrm{n}=37)$. A média das idades foi de 69,15 anos $(\mathrm{Q} 2=69, \mathrm{DP}=6,49)$, de escolaridade foi de 3,09 anos ( $\mathrm{Q} 2=3, \mathrm{DP}=2,81)$ e da renda familiar foi de $\mathrm{R} \$ 2.390$ ( $\mathrm{Q} 2=2.090, \mathrm{DP}=1.163)$. A maioria era casada $(n=61,93,8 \%)$ e cuidava de seu cônjuge $(n=58,89,2 \%)$. No grupo de cuidadores com boa/muito boa qualidade do sono $(\mathrm{n}=43)$, o escore de sobrecarga médio foi de 22,05 pontos ( $\mathrm{Q} 2=20,0, \mathrm{DP}=14,52)$ e, no grupo com qualidade do sono ruim/muito ruim ( $\mathrm{n}=22)$, de 32,77 pontos $(\mathrm{Q} 2=29,5, \mathrm{DP}=17,06)$. As análises comparativas evidenciaram diferenças significativas entre os grupos ( $U=285,00 ; p=0,009)$. Conclusão: Conclui-se que existe associação entre sobrecarga e qualidade subjetiva do sono em idosos cuidadores de idosos.

Palavras-chave: Cuidadores; Sono; Esgotamento Psicológico.

Agradecimentos: Conselho Nacional de Desenvolvimento Científico e Tecnológico (CNPq) e Fundação de Amparo à Pesquisa do Estado de São Paulo (FAPESP). 